Amazonía Peruana, Volumen XVII, №34, 2021; pp. 149-164

\title{
NARRATIVA DE LOS SERES ESPIRITUALES: COSMOVISIÓN Y CULTURA ASHÁNINKA
}

\author{
Beatriz Umaña Chiricente ${ }^{1}$ \\ Universidad Nacional Mayor de San Marcos-EILA \\ beatrizumana@unmsm.edu.pe \\ (1) https://orcid.org/0000-0002-8885-0717
}

\section{Resumen}

El artículo presenta la narrativa de los seres espirituales: cosmovisión y cultura asháninka, se centrará en la cosmovisión desde la cultura asháninka, clasificación de seres espirituales, la enseñanza que da la narrativa en la cultura asháninka. Recopilar información sobre la cosmovisión del mundo, cómo están clasificados los seres espirituales que viven en la tierra y cómo la narrativa de los seres espirituales hacen aporte a la educación desde la cultura. En la cultura asháninka el mundo está divido por etapas, cada ser cumple la función de cuidar y proteger a la humanidad y a la naturaleza. Estos seres son clasificados por ser dueños en maninakari, ser bueno protector y que vive en el cerro, y anakotiriri maninkari, los que pretenden ser mejor. Se ha investigado en kiatsi, mironi,

1 Este trabajo forma parte de la investigación de mi tesis de maestría en EIB del Posgrado de Letras, Facultad de Letras y Ciencias Humanas de la Universidad Nacional Mayor de San Marcos, cuyo asesor es el Dr. Gonzalo Espino Relucé; la que desarrollo como parte del grupo de investigación EILA-UNMSM. 
shashinti, kasonkati, kamari y ampatsi. Estos son algunos de los seres espirituales en la cultura asháninka. Estos conocimientos son recopilados en la comunidad de Cushiviani con el aporte de los sabios del distrito de Río Negro, provincia de Satipo, departamento de Junín.

Palabras claves: Comunidad; Narrativa; Seres espirituales; Cosmovisión; Cultura y asháninka

\section{Summary}

This article presents the narrative about Spiritual Beings: Worldview and Ashaninka Culture. It focuses on the classification of spiritual beings, the teachings expressed in the narratives of Ashaninka culture. It collects information about the worldview, how the spiritual beings living on earth are classified and how the narrative of the spiritual beings contributes to education based on the culture. In Ashaninka culture the world is divided into stages. Each being fulfills the function of caring for and protecting humanity and nature. These beings are classified as owners in Maninakari, good protectors and living in the hills and Anakotiriri Maninkari. Research was carried out on Kiatsi, Mironi, Shashinti, Kasonkati, Kamari and Ampatsi. It must be clarified that there are more spiritual beings in Ashaninka culture. This material was compiled in the community of Cushiviani with the contribution of the elders of the district of Rio Negro, Satipo province, Junin department.

Keywords: Community; Narrative; Spiritual Beings; Worldview; Ashaninka culture

\section{Introducción}

En esta investigación se aborda la narrativa de los seres espirituales en diálogo con mis vivencias y mi condición de mujer asháninka. La cosmovisión de nuestro mundo está dividida en: kibinti, kamabero, kipatsi y sharinkabeni y los seres espirituales viven en las cuatro dimensiones cumpliendo la función de cuidadores y protectores de la humanidad. Se trabajará sobre las características de los seres espirituales en especial, en los seres espirituales de la tierra: maninkari, ser bueno, y anakotiriri maninkari, que pretende ser mejor; así kiatsi, mironi, shashinti, kasonkati, kamari y ampatsi son seres negativos. Este estudio se hará a partir de los relatos y enseñanzas transmitidas por los abuelos. 


\section{Cosmovisión desde la cultura asháninka}

La cultura asháninka está llena de conocimientos, generalmente está relacionada con los seres espirituales, las actividades cotidianas que realizan los asháninka, el respeto y el pensar de nuestro mundo. Sanches-Chabarria (2012) afirma sobre la construcción del mundo (la cosmovisión) que todos los pueblos poseen una cosmovisión que satisface la necesidad de orientación y posicionamiento en la realidad. Las formas de conceptuar el cosmos, y la relación de este con el ser humano, son un producto cultural, socialmente aprendido. Cada cultura da por sentada las razones por las que las cosas son como son, señalando las bases legítimas del orden social. La comunera Violeta Ramos, nos dice: "La cultura es lo que somos, lo que hacemos, nuestra forma de vida, el respeto entre nosotros, respeto con la naturaleza, compartir conocimiento, entender y comprender la realidad de cosmos" (entrevista a Ramos Jari, 2020). Respecto a lo mencionado líneas arriba, se puede afirmar que cultura la forman las costumbres nuestras que están circulando en los espacios de los seres visibles e invisibles existentes en nuestro mundo. Para nosotros cultura es todo lo que tenemos: territorio, alimentos, medicinas, educación y a los dueños de la naturaleza. Es decir, todo lo bueno que hacemos, nuestra convivencia en la familia, comunidad y toda la sociedad a partir de las normas establecidas, el respeto de las personas y el bosque.

La cosmovisión asháninka se diferencia de las otras, en la libertad de conservar su conocimiento, sus propias creencias; por ello, en la actualidad se transmite mediante el aprendizaje y la práctica de manera individual y colectiva. Estos conocimientos están relacionados con los dueños de la naturaleza y transmitidos por los abuelos. La cosmovisión indígena, según el diálogo con Ferran Cabrero, "desvía la atención de la discusión liberal clásica del individuo y la colectividad (como simple agregado contractual) hacia la persona y la comunidad (como lugar durable, profundo y a la vez espiritual)" (2013: 67). En concordancia con Ferran, se puede afirmar que las cosmovisiones indígenas tienen una libertad de expresión, una manera de pensar o interpretar individual y colectiva, resulta verdadero que la comunidad es el centro del conocimiento que siempre se piensa en los seres sagrados existentes en nuestro mundo.

En la cosmovisión del mundo, en la cultura asháninka, la conexión entre nosotros y la naturaleza, están en relación y respeto a los atsipayetari kari añayeteri, es decir, a los seres invisibles y visibles designados por el ser divino convertido en humano. Cada uno de estos dueños cumple la función de cuidar y conservar la naturaleza y el mundo. Se puede decir que es un aprendizaje desde niños; y, 
en esa relación diaria entendemos el mundo de manera individual y colectiva: son conocimientos que vamos practicando y transmitiendo.

\section{Los mundos de la cultura, nuestra cosmovisión asháninka}

Nuestra memoria dialoga con las producciones escritas sobre mi cultura. El mundo resulta un espacio inmenso donde habitan personas, atiripe, animales, pirantsipe, vertebrados e invertebrados (reptiles, anfibio, aves, peces, insectos, sanguijuelas, lombrices y arácnidos, etc.); seres vivos y muertos convertidos en animales peyaripe, los seres espirituales buenos y malos existentes en la tierra y en los cosmos, en el mundo tierra y mundo de los seres divinos: en ambos hay aire, agua y plantas.

Nuestro universo comprende cuatro mundos. Mundo uno kibinti, mundo dos kamabero, mundo tres kipatsi, mundo cuatro sharinkabeni.

Conocer el mundo asháninka ayudará a entender dónde se encuentran los seres espirituales. El primer mundo Kibinti es el ser que nos mira desde arriba, el lugar donde vive está cubierto de nubes, tiene poder infinito; tal es así que todo los seres espirituales o dueños designados a cuidar y proteger, le temen. El segundo mundo, kamabero, es el lugar donde llegan los muertos, la dueña de muertos los identifica; si fueron buenos en la tierra, ellos se quedan con la dueña y si los muertos tenían actitudes negativas, son regresados al mundo de la oscuridad, sharinkabeni. El pueblo asháninka configura su noción de territorio en los mundos sharinkabeni y enokiiteni, que entrelazan sus espacios de interacción armoniosa entre el mundo de arriba y el mundo de abajo (Sanches-Chabarria 2012: 40). Para poder llegar a la oscuridad bajan en parinaro, algo como un tobogán que está situado en el río Tambo. En el tercer mundo, kipatsi, habitan los seres visibles e invisibles: humanos, animales, etc. Los peyaripe son los sheripiaripe que no cumplen con las dietas y prohibiciones; terminan convirtiéndose en seres mitad humano y animal. Existe muertos peyaripe, ellos son convertidos en animales, aquellos que no murieron por enfermedad. E1 cuarto mundo lo conocemos como sharinkabeni, la oscuridad, donde viven los demonios ciegos que conocemos como kamari, las personas que no fueron buenas en vida son enviadas a este mundo.

Los primeros habitantes en la tierra son los seres espirituales designados a ser dueños del río, de los árboles, de los animales, las plantas medicinales, etc. Luego los humanos son traídos a la tierra del lugar sagrado donde viven los seres buenos; por ser incrédulos y desobedientes, viene abireri a la tierra para 
controlar sus actos negativos; el castigo que recibieron es ser convertidos en animales cuyo comportamiento es muy parecido al comportamiento animal.

Existe relatos sobre la existencia de los seres espirituales: "Según los relatos, nuestros ancestros fueron creados por el dios Sol y la madre Luna. Se dice que antiguamente todas las estrellas que vemos hoy, eran personas que vivían aquí en esta tierra, pero esas estrellas no han podido volver a la tierra de origen porque no saben cómo regresar y, por eso, se quedaron allá arriba, en Oitaque, lugar donde moran nuestros antepasados". (Casanto 2008). Entre nosotros también contamos que Kashiri y Paba nacieron en la tierra. Cuando nació Kashiri, su madre murió. Kashiri era de piel blanca, lloraba muy fuerte, su llanto era insoportable y hacía doler el oído; por eso Nabiri lo envió al cielo. En otras narraciones el Sol y la Luna nacieron en la tierra, Paba es enviado al cielo porque nació excesivamente caliente y su madre murió.

En la entrevista con algunos sabios como Benigno Vicente Nicolás (2021), este agrega un mundo más. Lo llama ñateni, entendido como el mundo del agua. Dentro del agua están todos los seres vivos acuáticos existentes (peces, anfibios, moluscos etc.) que, dicho sea de paso, son fuente alimenticia para la existencia de la vida humana en la tierra.

Para nosotros los ashéninka todas las criaturas acuáticas existentes y están bajo el control del noonki (boa) consideradas como la dueña de estos seres vivos. El noonki crea, protege, y cuida a sus criaturas para que no sean exterminadas por el hombre. El pescar de manera constante e irracional causa molestia y cólera al noonki; entonces, provoca reacción inmediata dañando al espíritu del hombre hasta conseguir su muerte. (Entrevista a Vicente Nicólas, 2020)

Esta diferencia se produce porque existen diversas versiones de tradición oral del asháninka y ashéninka, así como el aporte sobre un nuevo mundo ñateni: lugar de los seres espirituales protectores de las especies existentes en el río. Acorde a las investigaciones realizadas sobre ñateni, este vive en el mundo tierra, su domicilio es en el río, este es designado como dueño de los peces que tiene un parentesco con mapichiri serpiente de piel briosa con diversos diseños. Esta serpiente es respetada por la cultura shipibo, estos mencionan que de ahí aprendieron sus diseños de tejido. Casanto (2014) afirma:

Tenemos el mundo del agua, donde está el sistema de los peces. Nosotros sostenemos que dentro del agua hay un ser considerado como la dueña: la boa, creadora de los peces, alimento importante para el ser humano. Pero no solamente para el ser humano, también para los peces mayores que se alimentan del pez menor, además tenemos a las aves y otros animales cuya 
fuente de alimentación son los peces. Por eso, en el mundo del agua tenemos que ver quién va a alimentar a quién. Tenemos un productor y un consumidor. En el mundo de la tierra existen animales, plantas, aves e insectos. Está el otorongo, que se considera el dueño. También hay unos seres que son mitad persona y mitad animal. Ustedes dirán ¿por qué? Podemos decir que es debido a una maldición. Si las personas no respetan las normas y cometen un error, entonces son castigados de esa forma, por sus malos actos. Nosotros manifestamos que el tabaquero asháninka ve todo este sistema de la tierra, mientras que el ayahuasquero ve todo el sistema del agua. Hay una conexión entre el ayahuasquero y el tabaquero. El tercer mundo es el cielo de los espíritus de los muertos, donde están los malos espíritus que esperan el proceso de calificación para ser designados después de la muerte. El cuarto mundo es el cielo de los astros, que son seres humanos convertidos en astros. Cada astro tiene su propia historia sobre cómo se convirtió y en qué tiempo del año aparece. (Casanto Shingari 2014: 239-240)

Casanto describe cuatro mundos; también explica sobre el primer mundo del agua considerando a la boa como dueña. Mayor y Bodmer (2009: 134) señalan que el mundo está sostenido por dos ejes. Uno de ellos fue puesto por el dios Sol para que no vuele la tierra, y que toda la gente esté tranquila. Sumado a ello él puso los cerros Intatoni y Antamaraka, rocas sólidas por donde sale al agua para regar toda la faz de la tierra. En el otro eje, por donde se hunde el Sol, están los cerros Omoro y Otsiriko. Los que sostienen estos ejes por debajo, para que la tierra no vuele, son seres invisibles que se llaman Nabieri (debajo del cerro Omoro) y Pachakama (debajo del cerro Otsiriko). Sosteniendo el otro eje están otros dos seres poderosos que se llaman Inkari (debajo del cerro Intatoni) e Inkami (debajo del cerro Antamaraka). Estos seres cuidan la entrada y salida del agua. Si ellos descansan, la tierra se ahogaría en pleno caos por su desobediencia y juego se quedó sosteniendo a nuestro mundo, si baja su mano produciría un caos en la tierra la destrucción.

\section{Los seres espirituales}

Los seres espirituales son los seres a quienes difícilmente se les ve, la persona que con facilidad interactúa para ser guiado y poder enseñar sus conocimientos es el sheripiari mediante la toma de ayahuasca. Las personas incrédulas y personas que en exceso cazan, recolectan frutas, gusanos comestibles y no comparten lo que obtiene en el bosque, a veces pueden ver a estos personajes míticos. Estos seres espirituales son dueños designados por el ser divino convertido en humano que cada uno cuida y protege; su castigo es hacerse visible y el llamado de 
atención sobre el acto cometido. El intruso puede ser agredido y solo sobrevivirá si es curado a tiempo por el sheripiari. Estos dueños viven en el aire, la tierra, la oscuridad y el cielo. Los seres espirituales se dividen en dos: buenos y malos. Maninkari que vive y cuida el bosque es bueno y los anakotiriri maninkari son los seres malos; siempre se adelanta a dar los castigos, siempre quiere ganar: maninkari se presenta para dar castigo a la persona que logra verlo, hace que enfermen o se mueran.

En el caso del mundo indígena, los relatos míticos afirman la existencia de espíritus y fuerzas superiores que controlan y determinan, de alguna manera, la vida de las personas. La tradición oral, muestra reiteradamente diversas afinidades y vínculos entre los seres de poder y los seres humanos, en una suerte de homologación, que se explica mediante recursos simbólicos como las relaciones de parentesco, las jerarquizaciones, los movimientos cíclicos o espirales, los ritmos, las emociones, los deseos, entre otros. Los seres de poder, astrales, terrenales o subterráneos y subacuáticos son considerados sociales, ya que establecen alianzas con los humanos, otorgan dones, castigan, combaten y experimentan necesidades diversas". (López Flores y Cuglievan 2012: 19)

Las afirmaciones de la existencia de los relatos de seres espirituales son enseñadas mediante la tradición oral tal como lo he confirmado en mi comunidad y al mismo tiempo registran diversos investigadores (López, Fernández) que estos seres tienen un vínculo entre Atsipayetari kari añayeteri seres buenos, malos y las personas. Estos seres son designados a cuidar y proteger en la tierra, aire, agua, mundo de la oscuridad, otorgan dones así como los conocimientos (curaciones, predicciones), castigan presentándose, peleando, proveen animales, frutas, plantas medicinales, árboles, maderables. Entre las expresiones míticas y transformación cultural se encontró que "los seres de la naturaleza se perciben desde el status de humanidad, mas no de especie; es el animal y los vegetales quienes se encuadran en muchos casos deontológicamente en relación a los humanos" (Fernando y Dávalos 2020: 204). investigadores demuestran que estas cualidades y actividades se logran especialmente a través del mundo de los sueños y las premoniciones

Las diferentes transformaciones y transmutaciones antropozoomórficas se materializan en el ámbito social y son confrontadas en el campo del parentesco reconstituyéndose dentro del mito y la realidad, a través del chamán como árbitro de los diferentes espacios de su mundo. Muchos ayllus kichwa se identifican con un antepasado pariente animal y chamán, rasgo mítico que también se ha retractado en las representaciones modernas de la y el sujeto kichwa. En la mitológica de este pueblo están presentes héroes culturales zápara lo cual se 
explica a partir del proceso de transculturación y etnogénesis que ha subsumido la cultura zápara en la kichwa. (2020: 204)

Los dueños de los animales, plantas, rocas tienen la facilidad de convertirse en el ser que quiere para proteger a los que le han designado.

Para saber dónde vivimos acudimos a los relatos narrados por nuestros ancestros. Cuando estamos vivos estamos en Kipatsi, cuando estamos muertos podemos vivir en el mundo de Kamabero donde se encuentran las personas buenas y Sharinkabeni a donde los muertos son enviados, al mundo de la oscuridad. Los seres espirituales viven en la tierra, cielo, aire, oscuridad, pero nos enfocamos en algunos seres espirituales que viven en la tierra, Maninkarite y Anakotiriri Maninkari que son Kasonkati, Shashinti, Mironi, Kiatsi, Ampatsi sabemos dónde vivimos y dónde viven los seres espirituales por medio de los relatos. Según Espinosa (2017: 63), estos relatos expresan, también, la manera de ver el mundo, la naturaleza, las personas y otros seres. Hablan de la época de los orígenes, así como del orden del mundo y la relación entre distintos seres", concuerdo con Espinosa, los relatos transmiten todos nuestros saberes de nuestra existencia y los que viven en la tierra, aire en el más allá.

\section{Clasificación de seres espirituales}

Los seres espirituales son clasificados como protectores y designados. Los protectores cuidan el agua, roca, animales, plantas medicinales, árbol maderable, ojo de agua y caracoles. Los designados cuidan a un solo ser: puede ser una planta medicinal, agua, árboles maderables, frutas, gusanos, entre otros. Según nuestra investigación, los seres espirituales de la tierra están clasificados por los lugares donde viven, otishi, nija, imperita y tampia. El ser Kamari vive en el aire y Sharinkabeni es conocido como el diablo más poderoso y más temido por los asháninkas; el castigo por no cumplir el horario para caminar, no se debe andar en la noche, cuando hay ventarrón porque el Diablo te puede llevar. Cuando hacemos mal, nos portamos mal, al final de la vida nos lleva el malo, el Kamari. Por eso no hay que pecar... el kamari. Pero hay veces que nos visita el kamari. 'tamos como ahorita conversando y escuchas, lo escuchas, ahí 'ta, por un susto también puede ser que te dé... (Martel 2009: 75) 
Clasificación de los seres espirituales

Los seres espirituales son clasificados por ser protector y designados

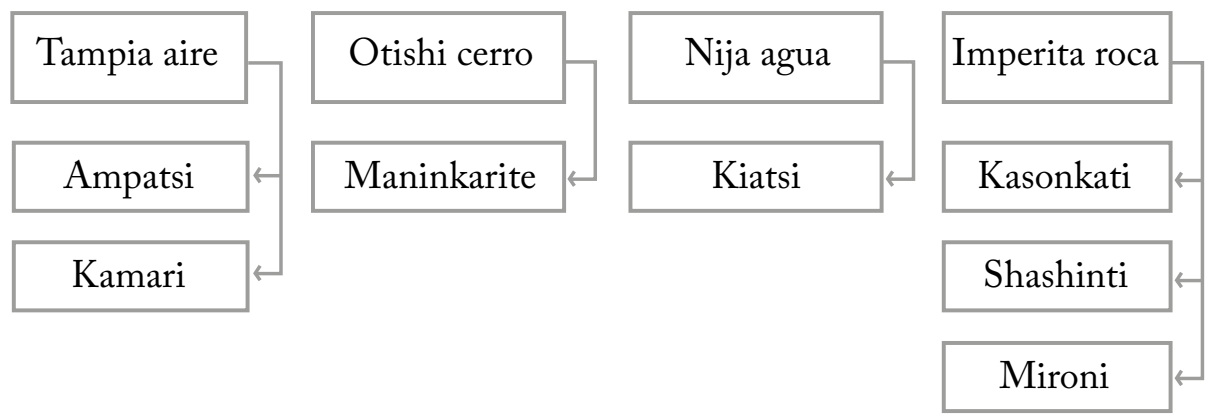

\section{Kasonkati}

Itimabeitani perani kasonkati osheki yoimintsarobantini, aka anampiki Cohiviaki itimini kasonkati ishiabetari boro irotya ipotsoti koronkari, kitamarori jero kisari, omoronta iyerito.

Aparo kitaiteri ijajeti ashaninka yameni tsoiroki, sankiro, mapoto antamiki, okanti tsinane eiro pitsarajetapaitsi, pimajerejetape chora ashitariri yoka poshinirintsi, kemetachari te ikemisantajeite chenkaberipe, ari itsarajeiti, itsinampabaka, kematiri tsibani ikitenkake kiteee, kiteee, kiteee.

Tsinane okemake ikitenkake tsibani okantajetajiri pishintsijetaje tsame ajataje kitenkanake, chenkaberi ikantajeti jeei jeei, jojojo, jojojo, te añeri kasonkati eiro pitsarobitsi, ari amonkoti tsinane otakero, okantanake tsamajatyeri okemantajetanakari janchinnn janchinnn janchinnn, e okantanake koraketake ashitariri akerika.

Okantanake tsenkabero te nomatanajia nariabetanaka chora intakotajarori ishitikanakero otsakiki itininkajiro te añanajero oshintsinka, ari ishiajetaja irosati yoijampoiretakero kasonkati, iro tikabakeri nija marojeini omontyanajetanakero tsako. Tsako, tsako, ari isaikanake mironi ikajemabeti janchinnn janchinnn janchinnn, jee itiritanakeri sankiro iyoche.

Ijajebetaka shiramparipe te iñapajeri ikanti isheripiari meka pijajetakerika eiro pitsenkabetapaitsi, kametsa pijajete pikisakakari ashitariri sankiro, yakempime ari itsijetakempime. (Chiricente Mahuanca L. 2019) 


\section{Kasonkati}

Antes kasonkati hacia asustar, en nuestra comunidad Cushiviani existía kasonkati es parecido al burro con franjas de color blanco y negro tenía la rodilla hueca, un día un grupo de asháninka fueron a recolectar caracol mediano y grande en el cerro, dijo la mujer no deben hacer ruido tiene su dueño lo que vamos a recolectar, empezaron hacer bulla, hacer bromas se escuchó silbido del chicua kiteee, kiteee, kiteee.

La mujer escuchó el silbido del chicua les dijo apúrense vamos a silbado, los incrédulos se han reido jeei jeei, jojojo, jojojo, no hay no tengan miedo la mujer boleado coca le quemo, tenemos que irnos, de un de repente escucharon janchinnn janchinnn janchinnn, eeee dijo ya viene el dueño lo que estamos recolectando.

Dijo la incrédula ya no puedo y empezó a echarse. Hubo uno que le amarró en su cintura y le empezó jalar, ya no tenía fuerza, entonces escaparon, y el kasonkati seguía sus rastros, el agua le separó y todos cruzaron el agua tsako, tsako, tsako, hay se quedó kasonkati empezó a rebuznar janchinnn janchinnn janchinnn, hooo le embarró su esperma al caracol.

Los hombres fueron no lo encontraron dijo el sheripairi ahora cunado van no deben ser incrédulos vallen con fe no hagan renegar al dueño del caracol, si te atrapaba les hubiera violado.

\section{Maninkarite}

Perani oshekini poshinirintsi te akoityabetyaji keme meka arosati kantakaro, itimi ebankari kari kemisantatsiri ijapinti otishiki ikobintsayeti osheki, mana ikantebetari eriro pipiapiatatsi chora ashitariri ari ishemakiampi ariya yoinijakakiampi.

Ipitanaka ebankari otishiki ikentabeti tsamiri ari nianki ikitenkakeri tsibani te ikemisanteri aikero ijasanoti otishiki, yaretapaka ipankoshitapake iñantabakariri shintori ikentabake, ipokitapa osheki poshinirintsi iyejiyetaka te imatabaje ikentanaje.

Inantabakari poshinirintsipe yatiriyetanake ikantiri ebankari paita pikobintsantantari osheki poshinirintsi ari itsarobanake, ikamantanake ipokapake ashisanotarori otishi maninkari amenakoyetiri ashaninkape jero otishi, itasonkajiri ikantabajiri pijataje pikamantajeitapajeri kobintsaripe eiro yaparayetiri poshinirintsi timatsi ashiyetarori. Ari yaretaja ibankoki majirei 
ijokitapaka, iyotabakeri sheripiari iñakakari maninkari aisati yatiritimotakari poshinnirintsi, Ikanta yabisakotaji ebankari ikishoketabajiri. (Cueva Mantari M., Maninkarite 2019)

\section{Maninkarite}

Antes existía muchos animales no era escaso como ahora nosotros somos los culpables, había un joven que no hacía caso siempre iba al bosque a cazar en exceso, a pesar que le decían no vayas todos los días, tiene dueño se cansará se te aparecerá.

Regresó el joven al bosque a flechar paujil en el camino silbó chicua. No le hizo caso, se fue más lejos, al llegar hizo su choza vio a un sajino, le flechó, empezaron a venir muchos animales gordos, no pudo flechar.

Empezó a ver los animales convirtiéndose en persona, le dijo joven porque cazas en exceso a los animales. En ese momento se asustó, vino el dueño del bosque maninkari el protector de las personas y lo existente del bosque, le sopló y le dijo que comunique a los cazadores no cazar para desperdiciar animales porque tiene dueño. Entonces llegó a su casa, estuvo en silencio, se enfermó, el sheripiari sabía lo que le sucedió, se le apareció manikari y los animales se convirtieron en humano, le soplaron al joven y sano. (Cueva Mantari M. , Maninkarite 2019)

\section{Kiatsi}

Onebiatari kiatsi ebankari, itimini ebankari japinitatsiri nijaki ikitsate oshabinijitanajerika, te ikemisante ipitanaja nijaki, okantabetari iniro erio pijati onijaikari ashitarori nija ari nakemi eiro noñajimpi.

Aparo kitaiteri ijatake nijaki ari ipiaka, shabiniji yaretapaka yamapake osheki shima antaripe ikejepinkatanaka yapiniti shimasanori, isampiyetiri jaoka pikantiri pairi shima, naro nojabetya te nayeji nopiashitajia. Ari imajereti meka ipiaja abisake osheki kitaiteri te añajeri mana ikobayebetari tekatsi ñajerine.

Iñashiyetabajari ijokitapaka sheripiari isatabakeri ikantiri eiro pikamantitatsi paita abisakempiri pamatasanotabajiata, abisakoperotaji ebankari ikamantajiri pashinipe ebankari anakeri ashitarori nija kiatsi tsinane kamestaro anakeri tsompijaki opabetakari shima, parenti, kaniri te iroyaji, ikenkeshiriaja ikantetakeriri aririka akempi kiatsi eiro pibakotaro, te irobayetampe, ari obashiretakotajari kiatsi okantajiri chora aka aotsi pijataje ikenkeshiriaja 
ikenanake aotsi orijaniki ari yaretsapitapaji isokijantajari. Meka ikantiri sheripiari pikemisantaje meka ikantetempirika eiro pipiapiatatsi nijaki chora ashijatarori. (Chiricente Mahuanca L. 2019).

\section{Kiatsi}

Se enamoró kiatsi de un joven, había un joven que diario iba al río a anzoliar en las tardes, no hacía caso. Regresó al río, su madre le anticipó te puedo enseñar. La dueña del río te llevará, no te volveré a ver.

Un día se fue al río. Se perdió, en la tarde regresó y trajo muchos pescados grandes, siempre pescaba chupadora, le preguntan cómo obtienes pescado, cuando voy no pesco nada, regreso sin nada. Quedó en silencio, luego regresó, pasó muchos días no aparecía, todos le buscaron nadie volvió a verlo.

De la nada apareció y al llegar se enfermó el sheripiari, le bañó, le dijo no avises lo que te sucedió hasta que te recuperes. El joven sanó y le comunicó a los demás jóvenes que le llevó la dueña del rio kiatsi mujer linda, le llevó dentro del agua le dio pescado, plátano, yuca no comió, recordó lo que dijeron: cuando te lleva kiatsi no debes comer lo que te da. No comió, se entristeció kiatsi le dijo aquí hay camino, anda. Se recordó, se fue por el camino angosto, llegó a la orilla, así salió. Le dijo sheripiari tienes que obedecer. Cuando te recomiendan no debes responder, el río tiene dueña. (Chiricente Mahuanca L. 2019).

\section{Enseñanza y narrativa en la cultura asháninka}

Los abuelos son los principales narradores que transmiten los conocimientos y enseñanzas de los relatos sobre seres espirituales que terminan siendo ejemplos para el oyente (Marcos Quintimari 2020). Los relatos respecto a seres espirituales son transmitidos desde los abuelos y se continúa transmitiendo a los niños; es decir una lengua cultural de comunicación y transmisión de conocimientos y aprendizaje.

Los relatos transmiten conocimiento de la cultura sobre lo sucedido en nuestra historia. Estos relatos deben ser escuchados por los jóvenes porque son enseñanzas para proteger a la naturaleza y vivir feliz (Chiricente Mahuanca 2020).

El relato en la cultura asháninka es importante porque transmite los saberes culturales, la convivencia armónica de la naturaleza y la humanidad y permite reflexionar, reir o llorar con las historias; es decir, todo lo que existe en el mundo 
asháninka es conocido desde la tradición oral, lo que da perfil a nuestra identidad y continuidad a nuestra memoria colectiva, de allí la importancia que tiene la tradición oral:

Se trata de una estructura comunicativa que corresponde a formas tradicionales de transmisión del imaginario y memoria colectiva. Es decir, tipos de textos que se dicen en una determinada comunidad cultural, que los conoce y los tiene en su memoria. Estos tipos de relatos no son estáticos, por el contrario, sugieren siempre una densidad episódica de la que, eventualmente, se podría rastrear en su propia historia lo que va dejando y lo que va incorporando a lo largo del tiempo. (Espin 1988: t185)

Cada comunidad tiene su propia cosmovisión de cómo entender el mundo, los relatos fluyen en su dinámica armónica con el transcurso del tiempo.

La cultura posee conocimientos; en este caso, los seres espirituales han sido de vital importancia en la educación sobre la protección de la naturaleza. Con el trascurrir de los años se ha estado perdiendo el conocimiento, respeto y cuidado por la generación, asimismo los migrantes han destruido lugares sagrados para la agricultura.

\section{Conclusión}

La cosmovisión del mundo en la cultura asháninka se presenta en etapas: Kibinti, ser divino, protector de todo lo creado en la naturaleza; Kamabero, dueña de los muertos, lugar donde se identifica los muertos bueno y malos, además es donde permanecen los muertos buenos; Kipatsi donde habitan los seres visibles e invisibles, humanos, animales terrestres y acuáticos; maninkari ser protector de las personas y animales, frutas, provee alimentación. Además es conservador de la naturaleza, brinda conocimiento; y, Anakotiriri maninkari, seres que quieren ser mejores, hacen daño entre los buenos y malos; son dueños designados por el ser divino, otros autores lo consideran mundo del agua.

Los seres espirituales son invisibles y viven en el universo. Cada uno cumple una función de cuidar y proteger a la humanidad y naturaleza, quien tiene facilidad de verlo es el sheripiari, a quien le transmite conocimiento y enseñanza, también le comunica lo que va a pasar.

Los seres espirituales se clasifican como seres de aire y de tierra; en este caso nos centramos en algunos seres que viven en el mundo de la tierra Kiatsi, Mironi, Shashinti, Kasonkati, Kamari, Ampatsi y Maninkari. Están clasificados por ser dueños y lugar donde viven de roca, aire, agua, cerro. 
Las narrativas de seres espirituales son tomadas como ejemplos de enseñanza, la educación propia para la conservación de la naturaleza, que muestra el respeto a los seres invisibles y las personas, permite una buena convivencia en la familia y comunidad, además mantiene la memoria cultural. 


\section{Referencias bibliográficas}

Cabrero, Ferran. "Ejercer derechos, refundar el Estado. Cómo los indígenas amplían la ciudadanía”. En: Ciudadanía intercultural. Aportes desde la participación políticade los pueblos indígenas en latino américa. PNUD, Estados Unidos: 2013, 12-101. https:/www1.undp.org/content/undp/ es/home/librarypage/democratic-governance/Ciudadania-InterculturalAportes-desde-la-participacion-politica-de-los-pueblos-indigenas-deLatinoamerica.html.

Cabrero, Ferran (coordinador). Ciudadanía intercultural. aportes desde la participación politicade los pueblos indígenas en latino américa. PNUD, Estados Unidos: 2013. https://dds.cepal.org > redesoc > publicacion.

Casanto, Enrique. Poñaantsi. Relato sobre el orígen ashaninka. Proyecto Pueblos Indígenas y Desarrollo Sostenible, 2008.

Casanto, Enrique. "He pintado cuadros sobre cómo pueden" (versión PDF). En: Mundo amazónico. 2014,237-243. https://revistas.unal.edu.co〉 article> download.

Espino, Gonzalo. "Actos de habla en relatos de tradición oral: Notas sobre un relato oral cusqueño". En: Escritura y Pensamiento, 1(2). 1988. https:// revistasinvestigacion.unmsm.edu.pe/index.php/letras/article/view/6389

Espinosa, Óscar. Los pueblos shipibo-konibo, isconahua y kakataibo. Ministerios de cultura. Lima: 2017.

López, Carmen y Gisela Cuglievan. Asháninka: territorio, historia y cosmovisión. CILA, UNMSM. Lima: 2012.

Martel, Víctor. Cambios semánticos en el léxico relacionado a la cosmovisión ashaninka del Bajo Perené generados por el adoctrinamiento de la iglesia adventista. Tesis Licenciado en Lingüística. Facultad de Letras y Ciencias Humanas. Universidad Nacional Mayor de San Marcos, Lima: 2009. https://cybertesis.unmsm.edu.pe/bitstream/handle/20.500.12672/947/ Martel_pv\%28b\%29.pdf?sequence=1\&isAllowed=y.

Mayor, Pedro y Richard Bodmer. "Pueblos indígenas de la Amazonía peruana" (versión PDF). Iquitos-Perú: 09 de julio 2009. http://www.scielo.org.mx» scielo. 
Sánchez, Rodolfo y María Chavarría. "Investigación aplicada de la educación intercultural bilingue asháninka territorio, historia y cosmovisión”. Lima: Tarea, 2012.

Yale, Diego. "Cosmología y transformación en la percepción de los kichwalorocachi del pueblo ancestral Kawsay Sacha. Río Curaray. Amazonía ecuatoriana" (versión PDF). En: Revista de Creencias Sociales y Humanas La Universidad Politécnica Salesiana. 2020. https://dialnet.unirioja.es

\section{Entrevista}

Chiricente Mahuanca, L. A. "El relato tiene una historia". (B. O. Umaña Chiricente, Entrevistador) Perú, Satipo-CN Cushiviani, Junín, 02 de julio, 2020.

Marcos Quintimari,J. “Los relatos se transmite de generación en generación”. (B. O. Umaña Chiricente, entrevistador) Perú, Satipo- CN Cushivinai, Junín, 15 de julio, 2020.

Ramos Jari, Violeta (48), asháninka, natural de la comunidad nativa Cushiviani (Perú). Entrevista 9 de julio, 2020.

Ramos Jari, V. “La cosmovisión en nuestra cultura asháninka”. (B. O. Umaña Chiricente, entrevistador) Perú, Satipo-CN Cushivinai, Junín. 09 de julio, 2020

Vicente Nicolás, B. "La cosmovisión del mundo asháninka”. (B. O. Umaña Chiricente, Entrevistador) Perú-Pucallpa, 18 de febrero, 2020. 